\title{
GIANT ANEURYSMS:OUR REVIEW OVER 10 YEARS
}

\section{Dr Anindya Mukherjee}

\section{Dr Parthasarathi} Datta*

\section{Assistant Professor,anaesthesiology,nrs Med Coll Kolkata}

Associate Professor,neurosurgery,nrs Med Coll Kolkata. *Corresponding Author

ABSTRACT Giant intracranial aneurysms have a minimum diameter of at least $25 \mathrm{~mm}$.they represent $2-5 \%$ of all intracranial aneurysms and have a female preponderance .over a span of 10 years(january 2010 to january 2020) we operated 7 giant aneurysms and 45 small aneurysms.recent natural history studies have demonstrated an annual rupture rate for giant intracranial aneurysms(gia) to be around 6\% which is higher than for small aneurysms(1-3\%).we analyzed intraoperative rupture rate for giant aneurysms and compared with small aneurysms.the intraoperative rupture rate of gia is clinically less than small aneurysms but is not statistically significant. Conclusion:intraoperative rupture rate of gia is not more than small aneurysms statistically.

\section{KEYWORDS : GIANT,ANEURYSMS,INTRAOPERATIVE}

Gia have a minimum diameter of at least $25 \mathrm{~mm}$.giant aneurysms reppresent $2-5 \%{ }^{1-4}$ of all intracranial aneurysms and have a female preponderence.most patients become symptomatic in the fourth to sixth decade of life.

Hutchinson described the first giant intracranial aneurysm in $1875^{5}$.dandy was the first to actually expose and clip a cerebral aneurysm ${ }^{6}$.the morphology of giant aneurysms can be either fusiform or saccular.saccular aneurysms mostly occur at arterial bifurcations probably due to continuous hemodynamic stress.fusiform or dolichoectatic lesions may result from atherosclerosis,congenital arteriopathies or traumatic dissection.formation and rupture of aneurysms deppend on various factors like female sex , age , hypertension ,connective tissue disease and smoking.for a growing aneurysm, wall tension must increase to maintain the integrity of the wall.according to laplace's law, the stress over the aneurysmal wall increases in relation to the radius.but upto $60 \%$ of giant aneurysms have intraluminal thrombus which develops in areas where blood outside the turbulent flow stream stagnates, leading to thrombosis. whether gia are less prone for intraoperative rupture,remained the target of our study..although recent natural history studies have demonstrated an annual rupture rate for gia to be around $6 \%$ which is higher than for small aneurysms ${ }^{7,8}$.

\section{MATERIALS \& METHODS}

Over a span of 10 years(from january 2010 to january 2020) we have operated 7 giant aneurysms and 45 small aneurysms at our hospital.our inclusion criteria was :

1) single aneurysm

2) small and giant aneurysms

3) wfns grade 1 to 5

4) microsurgically clippped.

Patients were in the age 30 to 65 years.female $>$ male -preoperative evaluation was done after subarachnoid hemorrhage with 3d-ct angiogram brain with or without digital substraction angiography (dsa) cerebral vessels.post operative evaluation was done with ct-angio with or without dsa.anaesthesia protocol was same for all the patients.all the patients were managed by the same team of neurosurgeons and neuroanaesthetist.intraoperative rupture during dissection was noted.we compared the giant and small aneurysms in terms of their intraoperative rupture . minor bleeding and among the 45 small aneurysms 16 had intraoperative rupture. The result analysis did not reveal any significant difference in rupture rate of giant and small aneurysms.in our study aneurysms were more common in female (both small and giant aneurysms). although the rupture rate of giant aneunysms clinically looks insignificant but it did not become statistically significant.

\section{DISCUSSION}

Giant aneurysms are 2-5\%of all intracranial aneurysms. clinical features are subarachnoid hemorrhage and mass effect.in the anteror circulation the mass effect may be in the form of pain,visual acuity and field defect,external ophthalmoplegia,dementia.mental disturbances and epilepsy.in the posterior circulation multiple cranial nerve palsy, bulbar palsies and hemiperesis may be present.

Diagnosis is done by four vessel cerebral angiography,ct angiography,mri(to detect compressive effects on adjacent brain).

Pathological evaluation of giant aneurysms often shows lack of a muscular layer and degeneration of the elastic laminar layers.some authors have proposed that giant aneurysms grow from repeated intramural hemorrhage within the aneurysm wall,followed by thrombus formation and neovascularization.upto $60 \%$ of giant aneurysms have intraluminal thrombosis.intra-aneurysmal thrombus may develop in areas where blood outside the turbulent flow stagnates leading to thrombus formation.annual rupture rate of gia $(6 \%)$ is higher than for small aneurysms(1-3\%).in our series intraoperative rupture of giant aneurysms was not statistically different from small aneurysms.the factors which contributed to less intraoperative rupture of giant aneurysms are:

1) presence of multiple layers of thrombus within the aneurysm fundus reduces the effective diameter of the fundus.so according to laplace's law (tension on wall=internal pressure $\times$ radius), the effective radius decreases.this leads to decreased wall tension.

2) repeated intramural hemorrhage within the aneurysm wall leads to fibrosis. histopathological examination of the wall has confirmed the presence of fibrosis.this fibrosed wall is tougher than normal wall.

3) neck of the aneurysm being occupied by thrombus limits the ingress of blood into the aneurysm lumen.this leads to decreased internal pressure. 
rupture of giant aneurysms'.xianli et al calculated annual risk of rupture to be $11.5 \%$ per year .intraaneurysmal thrombus may result in aneurysm rupture through mechanical stretching.in contrast to giant aneurysms of the posterior circulation, none of the giant aneurysms of the anterior circulation ruptured ${ }^{9}$.

Chowdhury ,cappellani et al have analyzed preoperative ,intraoperative and postoperative factors leading to aneurysm rupture.according to them,large size and location in the posterior circulation are pre-operative factors leading to aneurysm rupture ${ }^{10}$.

Hakma et al in their study of 78 giant aneurysms and 250 small aneurysms, concluded that 'giant aneurysms in this series were less likely to present with subarachnoid hemorrhage $(46 \% \text { of giant lesions, } 83 \% \text { of smaller lesions) })^{11}$.

In the "clarity study"(clinical and anatomic results in the treatment of ruptured intracranial aneurysms) 782 patients with 782 ruptured aneurysms underwent endovascular treatment at 20 institutions.the conclusion was, the rate of intraoperative rupture is significantly affected by aneurysm location but not aneurysm size ${ }^{12}$.

\section{CONCLUSION}

Giant intracranial aneurysms are $2-5 \%$ of all intracranial aneurysms.annual rupture rate of giant intracranial aneurysms (6\%) is higher than for small aneurysms (1-3\%).in our study we tried to analyze the intraoperative rupture rate of giant and small aneurysms.we came to the conclusion, that intraoperative rupture rate of giant aneurysms is not statistically different from small aneurysms.

\section{REFERENCES}

1. Pia HW,Zierski J.Giant cerebral aneurysms:A review of clinical picture,diagnosis and management with illustrative cases.Neurosurg Rev 5:117-148,1982.

2. Weir B :Special aneurysms:Saccular.In Weir B(ed):Aneurysms affecting the nervous system.Baltimore,Williams \& Wilkins, 185-208,1978.

3. Shibuya M, Sugita K.Intracranial giant aneurysms.In Youmans JR(ed):Neurological Surgery:A comprehensive reference guide to the diagnosis and management of Neurological problems.Philadelphia,WB Saunders, 1310-1319,1996.

4. Onuma T,Suzuki J:Surgical treatment of giant intracranial aneurysms.J Neurosurg 51:33-36;1979.

5. Hutchinson J:Aneurysms of the internal carotid within the skull diagnosed 11 years before patient's death:Sponteneous cure.Trans Clin Soc(Land)8:127,1875

6. Laws ER Jr,Udvarhelyi GB:The genesis of Neuroscience by A.Earl Walker,MD.ppark Ridge III,American Association of Neurological Surgeons, 1998

7. The International study of unruptured Intracranial aneurysms investigators:Unruptured intracranial aneurysms-risk of rupture and risks of surgical intervention.N Engl J Med 339:1725-1733,1998.

8. Lawton MT Spetzler RF:Surgical management of giant intracranial aneurysms:Experience with 171 patients.Clin Neurosurg 42:245-266, 1995

9. Xianli Lv,Zhiyong Chen,Liguo Liu,Chuhan Jiang,Guihuai Wang,Jin Wang.Rupture of Intradural Giant Aneurysms:The Mode of Treatment,Anatoomical and Mechanical factors.Neurology India.67;11941199:2019.

10. Chowdhury T,Cappellani RB,Sandu N,Schaller B,Daya J.Perioperative variables contributing to the rupture of Intracranial aneurysm: An Update.The Scientific World J.2013 .Article ID 396404

11. Hakma Z,Ramaswamy R,Loftus CM.Mortality rates for giant aneurysms.Acta Neurochir.153;1621-1623:2011.

12. Pierot L,Cognard C,Anxionnat,Ricolfi F,CLARITY Investigators.Ruptured intracranial aneurysms:factors affecting the rate and outcome of endovascular treatment complications in a series of 782 patients(CLARITY study).Radiology.Sept;256(3):916-923:2010. 